\title{
The role of interlaboratory comparative tests/interlaboratory comparisons in the development of national, competitive products.
}

\author{
Ulzhan Kuleimenova \\ RSE "KazInMetr", Astana, Kazakhstan
}

\begin{abstract}
Annotation: Confidence in the quality of products, which were produced by the enterprise, is the key of success and competitiveness in the market. Confirming products' quality and environmental security of the manufacture is impossible without a well-functioning testing and calibration laboratories, which can provide the accuracy of their results.

Now, external evaluation of the laboratories' competence by proficiency testing is one of the main instruments of development of laboratories' activities and improving the quality of researches. Even with a perfectly functioning management system, high-precision expensive equipment and professional operators, without external evaluation laboratories can work for a long period with an offsets or significant random variations in measurement results. Only through proficiency testing, laboratories can reveal the presence of this kind of problem. Participation in Interlaboratory comparisons makes it possible to identify the "weak" sides of the laboratory operation and the successful implementation of preventive and corrective actions make them more "strong."
\end{abstract}

\section{Report:}

Interlaboratory comparative tests and comparisons (hereinafter - ILC) is a necessary and effective tool for ensuring the uniformity of measurements in the country and the quality of measurements in laboratories.

Interlaboratory comparative tests - the organization, implementation and evaluation of measurements or tests on the same or similar samples by two or more laboratories in accordance with the previously defined conditions. Interlaboratory comparisons - the comparison of the results of research, the metrological characteristics of measuring instruments. Proficiency testing - the assessment of the party's work on pre-established criteria through the ILC. [1]

The basic principles of the ILC are:

$\checkmark$ Voluntariness

$\checkmark$ Transparency

$\checkmark$ Competence

$\checkmark$ Independence

$\checkmark$ Privacy

$\checkmark$ Non-discrimination and the exclusion of the possibility of taking biased decisions. [2]

Confidence in the quality of products, which were produced by the enterprise, is the key of success and competitiveness in the market. Confirming products' quality and environmental security of the manufacture is impossible without a well-functioning testing and calibration laboratories (hereinafter $-\mathrm{TL} / \mathrm{CL}$ ), which can provide the accuracy of their results.

Nowadays, when the problem of testing quality system in the TL/ CL is especially acute, main attention must be focused on the accuracy and reliability of researches' results. One effective way to demonstrate the quality of the laboratories' results is to check it's qualification by the ILC.

ILC has great value for medical (clinical-diagnostic) laboratories, since correct diagnosis and treatment are largely dependent on the reliability of analyses obtained in the clinical-diagnostic laboratories. Reliability of analysis can be affected by several factors: accuracy of measuring devices, the appropriate preparation of the patient for analysis, proper execution of measurement techniques, as well as the correct interpretation of the results. In this regard, participation in proficiency testing rounds gives confidence to the laboratories by providing them with the accurate and reliable data.

Despite the increasing production of domestic products (CIS countries) and wide distribution them on the market, consumers still prefer to buy import goods, which were produced in the EU and the US territories. This is due to the high level of functioning of manufactures' quality systems in the above mentioned countries. One of the key factors of 
their production's success is the consumers' confidence in the activity of TL/CL. Accreditation System of European countries (Germany, UK, France, etc.) and the United States provide a high level of quality of the laboratories by ILC.

ILC enables to give an objective assessment of the reliability of tests, verifications/ calibrations conducted by laboratory and the results of its operations. In foreign countries, ILC has long been widely operating as one of the main tools of the laboratories' technical competence assessment. The number of proficiency testing programs (hereinafter - PTP) in Europe and the US is higher than their number in the CIS countries. According to the worldunified registry EPTIS and the web sites of respective countries' accreditation bodies Germany has 661 PTP, UK-280, USA - 299 while In Russian Federation-157, Belarus - 106, Kazkahstan-80. [3]

In addition, in abovementioned countries, issues of laboratory proficiency testing are regulated at the international level by the relevant regulatory and technical documents. The requirement of accreditation bodies on the participating in proficiency testing is based not only on the states of ISO / IEC 17025 "General requirements for the competence of testing and calibration laboratories". The International Organization for the accreditation of laboratories ILAC, as well as the European Association for Accreditation EA imposes their demands for the participation in the PTP and uses the results in practice, firstly, during the accreditation. One of the key document is the guide ILAC-P9: 06/2014 «ILAC Policy for Participation in Proficiency Testing Activities", which sets the guidelines for the accreditation bodies on using of proficiency testing in the process of accreditation of the laboratories. Under this policy, the technical competence of the laboratories can be demonstrated by successful participation in ILC organized with purposes other than proficiency testing, including:

- the evaluation of characteristics of test methods;

- the evaluation of characteristics of the standard samples;

- to compare the results of operations of two or more laboratories on their own initiative;

- as proof of the equivalence of measurements of National Metrology Institutes. [4]

Besides this, in the aforementioned manual are attached requirements for national accreditation bodies. One of these requirements is the presence of its own policy on the using the proficiency testing results.

Also, it should be noted the presence of unified worldwide registry of providers and PTP EPTIS (European proficiency testing informative system) (www.eptis.bam.de), the coordinator is the "Federal Institute for Materials Research and Testing» of Germany. Database EPTIS, which is aimed at accounting of regular functioning PTP, currently has about 3,000 PTP in the different areas from ILC providers of 40 countries.

As we can see, ILC is an integral part of ensuring the quality of production in Europe and America.

However, in CIS countries, ILC does not operate successfully enough, which can be noticed by the following facts:

- A small number of accredited and non-accredited providers;

- Narrow accreditation scope of ILC providers (lack of PTP);

- No unified online ILC resources (registry) in CIS.

Considering the increasing number of TL/CL in the CIS countries over the past decade, it is necessary to develop and pay more attention to proficiency testing procedure by ILC.

Let us consider the dynamics of the ILC development by the example of the Republican State Enterprise "Kazakhstan Institute of Metrology" (hereinafter - RSE "KazInMetr"). RSE "KazInMetr" is a State Scientific Metrological Center, which has acted as a provider of ILC since 2011. In 2014, Institute has accredited for compliance with the requirements of GOST ISO /IEC 17043 "Conformity assessment. Basic requirements for proficiency testing".

Today RSE "KazInMetr" - one of five institutions in the country, with the accreditation certificate, which confirms the international level of quality of performing work on the organization of ILC. 
In analyzing the ILC results for 2011-2016 years, the following figures have been identified:

\begin{tabular}{|l|l|l|l|l|}
\hline Year & $\begin{array}{l}\text { The number of } \\
\text { conducted ILC }\end{array}$ & $\begin{array}{l}\text { The number of participated } \\
\text { laboratories }\end{array}$ & $\begin{array}{l}\text { The number of } \\
\text { unsatisfactory } \\
\text { results }\end{array}$ \\
\cline { 3 - 5 } & & TL & CL & $15(21 \%)$ \\
\hline 2011 & 71 & 41 & 30 & $13(15 \%)$ \\
\hline 2012 & 85 & 45 & 40 & $10(10 \%)$ \\
\hline 2013 & 100 & 47 & 52 & $8(7 \%)$ \\
\hline 2014 & 120 & 50 & 70 & $8(7 \%)$ \\
\hline 2015 & 150 & 57 & 93 & $5(3 \%)$ \\
\hline 2016 & 200 & 65 & 135 & \\
\hline
\end{tabular}

Table 1. ILC of RSE "KazInMetr" for 2011-2016 years.

As we can see, there is a clear tendency to reduce erroneous results during ILC in 2016 compared to 2011. It indicates improvement of the quality level of laboratory researches and the positive experience of the work done by the organization ILC.

The most important and difficult task during the ILC is a statistical calculation of the participating laboratories' results and data analysis. However, it is important to carry out not only a statistical calculation, but also an analysis on metrological correctness and adequacy of the conducted measurements. Because the result also depends on the information provided by the participating laboratory (verification / calibration protocol, uncertainty report), the requirements and conditions performed during comparisons and the algorithm of statistical calculation.

Let's consider the results of ILC for the last 4 years of the provider RSE "KazInMetr"'s activity on calibrating a weight in $500 \mathrm{~g}$, accuracy class M1. Calibrations were conducted in accordance with OIML R 111-1 - 2009 "Weights of classes $E_{1}, E_{2}, F 1, F_{2}, M_{1}, M_{1-2}, M_{2}, M_{2-3}$ and $\mathrm{M}_{3}$. Metrological technical requirements" and Guideline 1747-87 "Measures of exemplary and general purpose masses. Method of verification". In these ILC programs, participating laboratories evaluated the actual weights and its corresponding uncertainty. As reference values were accepted the results of the calibration laboratory of the National Metrology Institute (RSE "KazInMetr"), obtained by the state standard of mass. Since the state standard of the mass' unit of the RSE "KazInMetr" was compared with the working standard of the International Bureau of Weights and Measures (BIPM), it provides international traceability of measurement results and is the most accurate in the country.

Figure 1 shows the results of the laboratories participated in ILC on calibration of weight for 2013-2016 years. In the round of comparisons took part 4 laboratories, indicated in figure from 2 to 5 . Number 1 indicates the assigned values obtained by the state standard. The green marks in the graph are real values, blue - the upper limits and red - the lower limits.

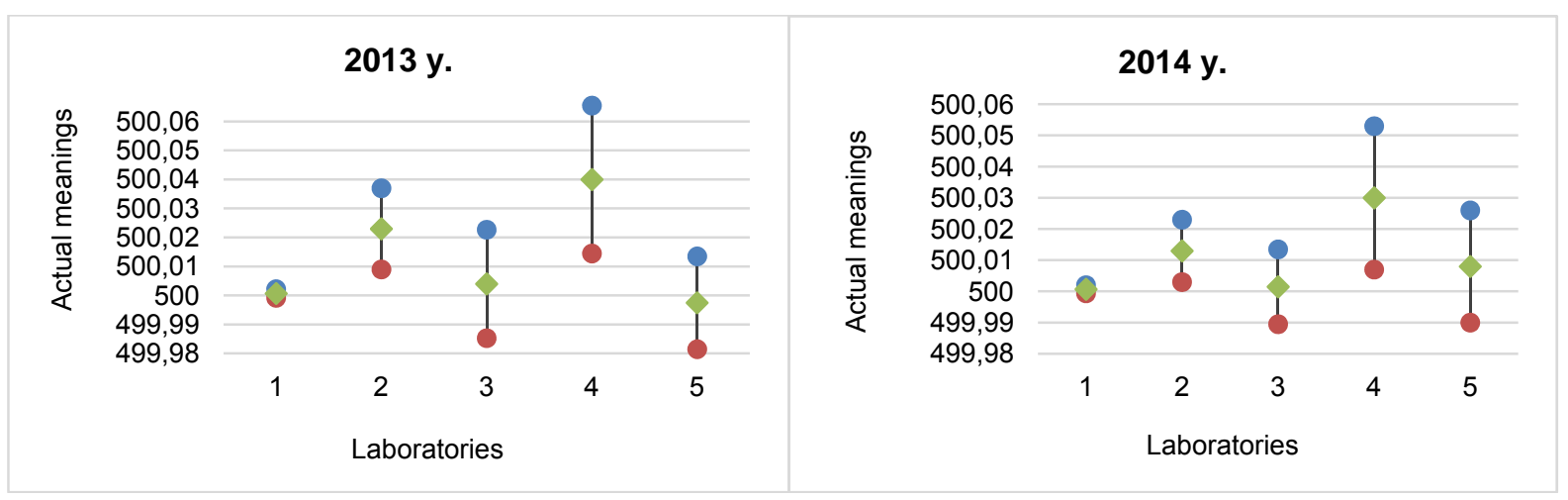




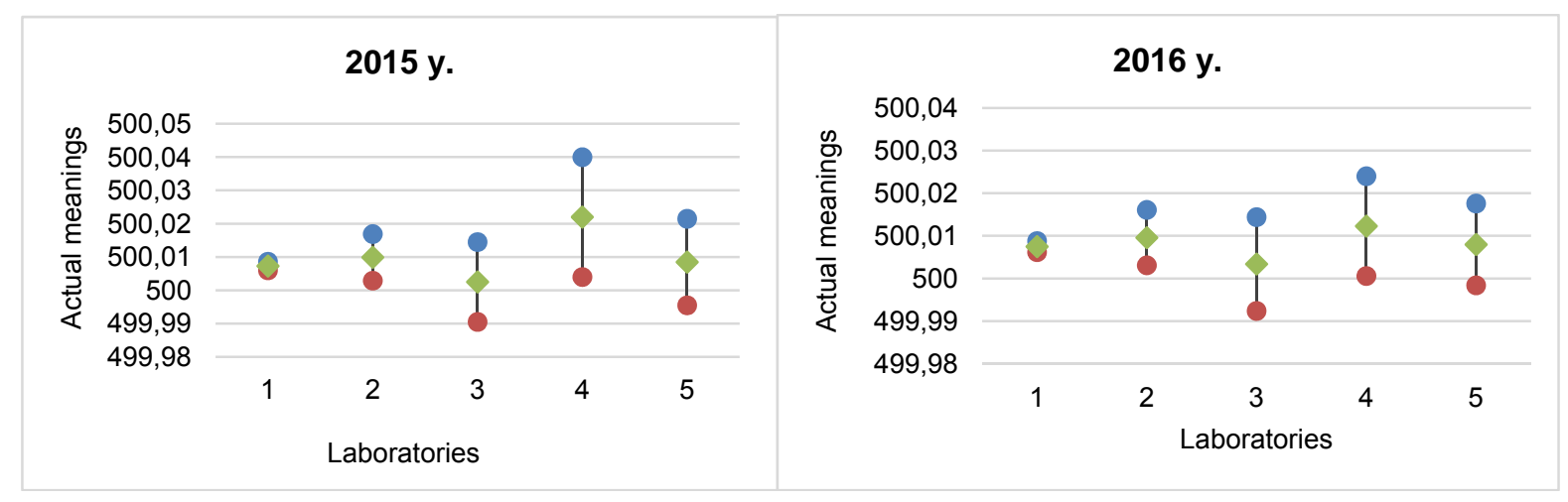

Figure 1. Data of ILC on weight calibration in 2013-2016 years.

Thus, it can be clearly seen from the graphs that the uncertainty values of the laboratories decreased year by year and stayed within the limits of the allowed (assigned) meanings. Which in turn shows increasing accuracy and correctness of the measurements. Through the analysis of errors and the organization of corrective measures, we have opportunity to improve technical activity of laboratories.

Now, external evaluation of the laboratories' competence by proficiency testing is one of the main instruments of development of laboratories' activities and improving the quality of researches. Even with a perfectly functioning management system, high-precision expensive equipment and professional operators, without external evaluation laboratories can work for a long period with an offsets or significant random variations in measurement results. Only through proficiency testing, laboratories can reveal the presence of this kind of problem. Participation in the ILC makes it possible to identify the "weak" sides of the laboratory operation and the successful implementation of preventive and corrective actions make them more "strong."

Advantages of ILC:

$\checkmark$ Improving the efficiency and the technical activity of laboratories;

$\checkmark$ A regular assessment of the quality of researches;

$\checkmark$ Validation and introduction of new methods / devices in laboratory practice, which in turn can be the basis for a new standard;

$\checkmark$ Professional development of personnel;

$\checkmark$ Increasing reputation in the domestic and foreign markets;

$\checkmark$ The advantage to customers and external regulatory organizations, including accreditation bodies and state supervisory authorities for the non-accredited laboratories;

$\checkmark$ Results of ILC $\rightarrow$ effective feedback $\rightarrow$ quality management.

Development of the activity of proficiency testing providers and ILC is the only key to the confidence and credibility of the TL/CL's work. Effectively functioning laboratories - the way of the domestic production and competitive products' success.

\section{Bibliography}

[1] GOST ISO / IEC 17043-2013 «Conformity assessment- Basic requirements for proficiency testing".

[2] www.stylab.ru

[3] www.eptis.bam.de, www.bsca.by,www.kca.gov.kg, www.proftestlab.ru, www.nca.kz

[4] "Providers of proficiency testing - The way of import substitution of services", S.V.Migan. Quality control of products №10-2015, 23-24 pages. 